\title{
BMJ Open Prospective evaluation of improving fluoroquinolone exposure using centralised therapeutic drug monitoring (TDM) in patients with tuberculosis (PERFECT): a study protocol of a prospective multicentre cohort study
}

Simone HJ van den Elsen, ${ }^{1}$ Marieke GG Sturkenboom, ${ }^{1}$ Onno Akkerman, ${ }^{2,3}$ Linda Barkane, ${ }^{4}$ Judith Bruchfeld, ${ }^{5,6}$ Geoffrey Eather, ${ }^{7}$ Scott K Heysell, ${ }^{8}$ Henadz Hurevich, ${ }^{9}$ Liga Kuksa, ${ }^{4}$ Heinke Kunst, ${ }^{10}$ Johanna Kuhlin, ${ }^{5,6}$ Katerina Manika, ${ }^{11}$ Charalampos Moschos, ${ }^{12}$ Stellah G Mpagama, ${ }^{13}$ Marcela Muñoz Torrico, ${ }^{14}$ Alena Skrahina, ${ }^{9}$ Giovanni Sotgiu, ${ }^{15}$ Marina Tadolini, ${ }^{16}$ Simon Tiberi, ${ }^{17}$ Francesca Volpato, ${ }^{16}$ Tjip S van der Werf, ${ }^{2,18}$ Malcolm R Wilson, ${ }^{7}$ Joaquin Zúñiga, ${ }^{19,20}$ Daan J Touw, ${ }^{1}$ Giovanni B Migliori, ${ }^{21}$

To cite: van den Elsen SHJ, Jan-Willem Alffenaar (iD) 1,22

Sturkenboom MGG,

Akkerman 0, et al. Prospective

evaluation of improving

fluoroquinolone exposure using centralised therapeutic drug monitoring (TDM) in patients with tuberculosis (PERFECT): a study protocol of a prospective multicentre cohort study. BMJ Open 2020;10:e035350. doi:10.1136/ bmjopen-2019-035350

- Prepublication history and additional material for this paper are available online. To view these files, please visit the journal online (http://dx.doi. org/10.1136/bmjopen-2019035350).

DJT, GBM and J-WA contributed equally.

Received 29 October 2019 Revised 06 March 2020 Accepted 21 May 2020

Check for updates

(C) Author(s) (or their employer(s)) 2020. Re-use permitted under CC BY-NC. No commercial re-use. See rights and permissions. Published by BMJ.

For numbered affiliations see end of article.

Correspondence to Dr Jan-Willem Alffenaar; j.w.c.alffenaar@umcg.nl

\section{ABSTRACT}

Introduction Global multidrug-resistant tuberculosis (MDR-TB) treatment success rates remain suboptimal. Highly active WHO group A drugs moxifloxacin and levofloxacin show intraindividual and interindividual pharmacokinetic variability which can cause low drug exposure. Therefore, therapeutic drug monitoring (TDM) of fluoroquinolones is recommended to personalise the drug dosage, aiming to prevent the development of drug resistance and optimise treatment. However, TDM is considered laborious and expensive, and the clinical benefit in MDR-TB has not been extensively studied. This observational multicentre study aims to determine the feasibility of centralised TDM and to investigate the impact of fluoroquinolone TDM on sputum conversion rates in patients with MDR-TB compared with historical controls. Methods and analysis Patients aged 18 years or older with sputum smear and culture-positive pulmonary MDRTB will be eligible for inclusion. Patients receiving TDM using a limited sampling strategy ( $t=0$ and $t=5$ hours) will be matched to historical controls without TDM in a 1:2 ratio. Sample analysis and dosing advice will be performed in a centralised laboratory. Centralised TDM will be considered feasible if $>80 \%$ of the dosing recommendations are returned within 7 days after sampling and $100 \%$ within 14 days. The number of patients who are sputum smear and culture-negative after 2 months of treatment will be determined in the prospective TDM group and will be compared with the control group without TDM to determine the impact of TDM.

Ethics and dissemination Ethical clearance was obtained by the ethical review committees of the 10 participating hospitals according to local procedures or
Strengths and limitations of this study

- To our knowledge, this is the first study that investigates the impact of fluoroquinolone therapeutic drug monitoring (TDM) on sputum smear and culture conversion rates in prospective patients with multidrugresistant tuberculosis (MDR-TB) versus historical controls without TDM.

- The feasibility for centralised TDM will be evaluated due to the participation of multiple healthcare centres located in differently-resourced countries from multiple regions in the world.

- The use of limited sampling strategies will reduce the burden of TDM for patients and healthcare providers while still providing a reliable estimation of drug exposure.

- A limitation is that this study focuses on TDM for moxifloxacin and levofloxacin only, being core drugs in MDR-TB treatment, without assessing other (core) anti-TB drugs.

is pending (online supplementary file 1). Patients will be included after obtaining written informed consent. We aim to publish the study results in a peer-reviewed journal. Trial registration number ClinicalTrials.gov Registry (NCT03409315).

\section{INTRODUCTION}

Tuberculosis (TB) is one of the major infectious diseases worldwide with an estimated number of 10 million new cases in 2017. ${ }^{1}$ In addition, multidrug-resistant TB (MDR-TB) 
remains a persistent problem with an estimated 458000 new patients in 2017. ${ }^{1}$ MDR-TB is treated from 9 to 20 months with a multidrug regimen. ${ }^{2}$ The grouping of second-line anti-TB drugs was revised in 2018 by the WHO. ${ }^{3}$ The fluoroquinolones, specifically moxifloxacin and levofloxacin, are now considered drugs of first choice (group A drugs), together with bedaquiline and linezolid, in the treatment of MDR-TB. ${ }^{23}$ The administration of group A medicines to patients with MDR-TB has been associated with increased treatment success and reduced mortality rates in comparison with other secondline anti-TB drugs. ${ }^{4}$ However, the estimated prevalence of fluoroquinolone resistance among MDR-TB cases is on the rise from $14.5 \%$ in 2011 to $22 \%$ in $2017 .{ }^{56}$ Mismanagement of MDR-TB treatment, especially the shorter regimen, could amplify the risk of drug resistance even further. ${ }^{7}$ Importantly, antibiotic resistance can be acquired due to non-compliance but also insufficient drug exposures (eg, interindividual pharmacokinetic variability in patients treated with fluoroquinolones).$^{8-11}$ Therapeutic drug monitoring (TDM) can help to prevent acquired resistance by individualising doses based on blood drug concentrations relative to the bacterial susceptibility, ideally measured as the minimal inhibitory concentration (MIC). ${ }^{712}$

Several studies described the role played by low drug concentrations on treatment outcomes. ${ }^{13-15}$ In the light of this evidence, it can be hypothesised that TDM, which aims for adequate dosing and exposure, could improve treatment outcomes. Yet, the added value of TDM in MDR-TB treatment outcomes has not been directly studied. ${ }^{16}{ }^{17}$ One retrospective study reported the effect of TDM on the treatment results of patients with drugsusceptible TB, either with and without diabetes. ${ }^{18}$ In the group without diabetes, TDM had a significant beneficial effect with $73 \%$ sputum culture conversion at 2 months among patients receiving TDM versus $60 \%$ in the control group. The positive effect of TDM was even larger in patients with diabetes and TB. The isoniazid or rifampicin dose was adjusted in 12 out of $17(71 \%)$ of the patients with diabetes based on peak concentration $\left(\mathrm{C}_{\max }\right)$ targets. However, this data is not available for the group without diabetes. To the best of our knowledge, such controlled studies have not yet been performed in people with MDR-TB.

The pharmacokinetic-pharmacodynamic parameter of fluoroquinolones is both time-dependent and concentration-dependent and therefore uses the ratio of area under the concentration-time curve (AUC) to MIC $\left(\mathrm{AUC}_{0-24} / \mathrm{MIC}\right)$. The target value is $\mathrm{AUC}_{0-24} / \mathrm{MIC}$ $>146$ for levofloxacin and free or unbound $\mathrm{AUC}_{0-24} /$ MIC $>53$ for moxifloxacin which corresponds to a total (bound and unbound) $\mathrm{AUC}_{0-24} / \mathrm{MIC}>106$ assuming a constant protein binding of $50 \% .{ }^{19}{ }^{20}$ However, multiple concentration measurements widely distributed over the dosing interval are required to compute the AUC from 0 to 24 hours $\left(\mathrm{AUC}_{0-24}\right)$. Limited sampling strategies (LSSs) could be adopted to reduce the burden of frequent sampling for both patient and personnel while providing a reliable estimation of $\mathrm{AUC}_{0-24}$ using only two blood samples. ${ }^{21} 22$

Unfortunately, TDM is not always easily accessible in high-TB-burden areas because of practical and financial reasons. Therefore, centralised TDM could be a valuable service. ${ }^{23}$ Large laboratories are generally well organised, have highly trained personnel with the adequate performance of analytical methods leading to reliable sample analysis results. ${ }^{24}$ In addition, centralising the TDM procedures will engender more consistent practice from healthcare practitioners familiar with TDM and the provision of dosing advice for anti-TB drugs.

The aim of the present study is: first, to investigate the feasibility of centralised TDM of moxifloxacin and levofloxacin in the treatment of MDR-TB recruited in TB reference centres located in different continents. Second, the impact of TDM on treatment results will be assessed by comparing 2 month sputum smear and culture conversion rates among patients who received TDM compared with matched historical controls without TDM.

\section{METHODS AND ANALYSIS Study design}

This observational, prospective, multicentre study aims to evaluate the feasibility of centralised TDM of moxifloxacin and levofloxacin as well as the impact of TDM on 2 month sputum smear and culture conversion rates of patients with MDR-TB. Study design and procedures are displayed in figure 1 . The study was registered at clinicaltrials.gov, recruitment started on 10 February 2018, and is expected to be completed in December 2020.

\section{Study location}

University Medical Center Groningen (UMCG) in Groningen, The Netherlands, is the coordinating centre and serves as the central laboratory facility for this study. The hospitals that are involved in patient recruitment are displayed in table 1.

\section{Study population}

Patients aged 18 years and older are eligible for inclusion if they are diagnosed with pulmonary MDR-TB, have positive sputum smear and culture samples at the time of inclusion, are treated with either oral moxifloxacin or levofloxacin, and provide written informed consent. Pregnant or breastfeeding women will be excluded. The decision whether a patient is treated with either moxifloxacin or levofloxacin is made by the clinician at the start of TB treatment based on local guidelines. Patients will not be actively assigned to use moxifloxacin or levofloxacin since this is an observational study.

A total number of 120 patients (60 with moxifloxacin and 60 with levofloxacin) will be prospectively included and compared with 240 matched historical controls (120 with moxifloxacin and 120 with levofloxacin). 


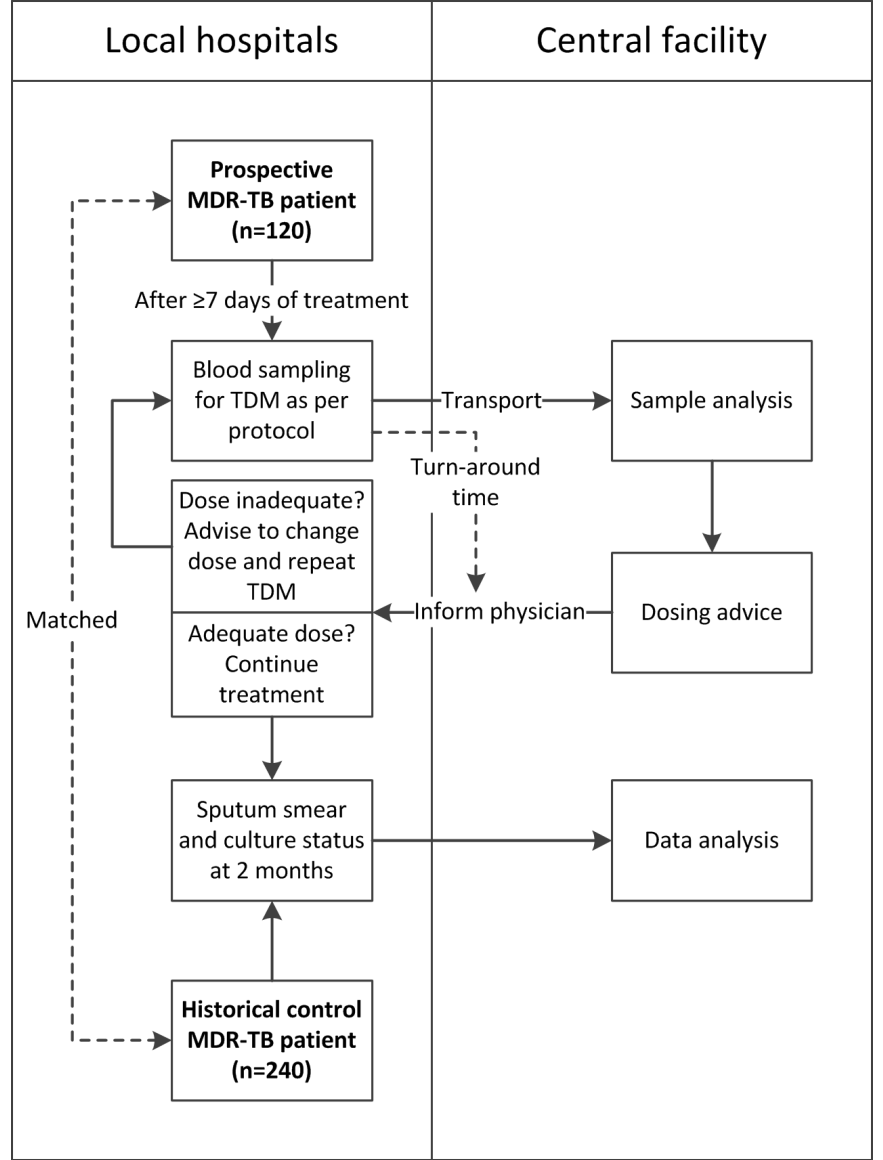

Figure 1 Workflow of study procedures in local hospitals and central laboratory facility. MDR-TB, multidrug-resistant tuberculosis; TDM, therapeutic drug monitoring.

Table 1 List of participating hospitals and their location

\begin{tabular}{ll} 
Hospital & Location \\
\hline University Medical Center & Groningen, The \\
Groningen (Central Lab Facility) & Netherlands
\end{tabular}

Tuberculosis Clinic "Beatrixoord", Haren, The Netherlands UMCG

\begin{tabular}{ll}
\hline Princess Alexandra Hospital & Brisbane, Australia \\
\hline Karolinska University Hospital & $\begin{array}{l}\text { Stockholm, Sweden } \\
\text { Mexico City, Mexico }\end{array}$ \\
\hline $\begin{array}{l}\text { Instituto Nacional de } \\
\text { Enfermedades Respiratorias }\end{array}$ & \\
\hline $\begin{array}{l}\text { Athens Chest Hospital "Sotiria” } \\
\text { Kibong'oto Infectious Diseases } \\
\text { Hospital }\end{array}$ & Athens, Greece \\
$\begin{array}{l}\text { Republican Scientific and } \\
\text { Practical Centre for Pulmonology, Tanzania } \\
\text { and Tuberculosis }\end{array}$ & Minsk, Belarus \\
\hline $\begin{array}{l}\text { Barts Health NHS Trust } \\
\text { St. Orsola-Malpighi Hospital, } \\
\text { University of Bologna }\end{array}$ & Bologna, Italy \\
$\begin{array}{l}\text { Riga East University Hospital TB } \\
\text { and Lung Disease Clinic }\end{array}$ & Riga, Latvia \\
\hline
\end{tabular}

NHS, National Health Service; TB, tuberculosis; UMCG, University Medical Center Groningen.
The following data will be collected in both groups: sex, age, body weight, height, country of birth, country of residence, comorbidities, QTc interval, laboratory values (kidney and liver function, electrolytes), history of previous TB treatment, bacterial susceptibility (including MIC if available), TB presentation (cavitary or noncavitary), current MDR-TB regimen (including drug dosages), sputum smear and culture data, treatment outcome (if known) and details on fluoroquinolone use (duration, possible drug interactions or adverse events).

Historical control patients will be matched on age, sex, Mycobacterium tuberculosis resistance pattern of the isolate (only regimen core drugs), comorbidities (HIV, diabetes and immunosuppression), presence or absence of cavitary TB on chest radiography and dosing of the fluoroquinolone $(\mathrm{mg} / \mathrm{kg}$ body weight, $\pm 10 \%)$ to prospectively enroled patients in a 2:1 ratio.

\section{Interventions}

The objective of the feasibility of centralised TDM will be assessed by evaluating the process, by which a locally collected sample will be analysed in a central laboratory and subsequent dosing advice will be returned to the local physician. In brief, after at least 7 days of treatment (steady state), two blood samples will be collected for TDM of moxifloxacin or levofloxacin according to a previously developed LSS. ${ }^{21}{ }^{22}$ The first sample will be collected just before drug intake $(\mathrm{t}=0)$ and the other at 5 hours after drug intake $(t=5)$. Samples will be transported to the central laboratory for drug analysis and will be accompanied by a form including key patient characteristics for personalised dosing advice (ie, sex, age, weight, height, serum creatinine, QTc interval, MIC, TB presentation, start of treatment, other anti-TB drugs and comorbidities). $\mathrm{AUC}_{0-24}$ will be calculated using a population pharmacokinetic model $^{21} 22$ and Bayesian dose optimisation in MWPharm++ (V.1.7.3; Mediware, Groningen, The Netherlands).

Dosing is optimised based on $\mathrm{AUC}_{0-24} / \mathrm{MIC}$ or $\mathrm{AUC}_{0-}$ ${ }_{24}$ (in case MIC is unknown), taking into consideration comorbidities (HIV, diabetes and immunosuppression), the persistence of TB symptoms and response to treatment so far. The Bayesian dosing software uses sex, age, height, weight and renal function in addition to drug dose and measured drug concentrations to forecast the drug exposure after a dose change. For patients who are at risk for treatment failure due to the previously mentioned reasons, a higher drug exposure is recommended. This is especially relevant in the case of an unknown individual MIC since the actual MIC might be near the breakpoint, to prevent treatment failure and acquired resistance. The target $\mathrm{AUC}_{0-24} / \mathrm{MIC}$ and $\mathrm{AUC}_{0-24}$ are shown in table 1 . If a dose change is necessary, TDM is to be repeated after at least 7 days after the initiation of the new dose (steady state). Dose increases of moxifloxacin will not be advised in the case of a prolonged QTc interval $(>450 \mathrm{~ms}$ for men and $>470 \mathrm{~ms}$ for women) because of safety reasons. As levofloxacin may be less cardiotoxic than moxifloxacin, 
levofloxacin dose increases are permitted in the case of prolonged QTc interval, but only with adequate electrocardiogram monitoring. Patients with prolonged QTC interval will not be excluded from the study since TDM can still be helpful to verify drug exposure. A closely monitored follow-up including MIC determination can be advised in the case of $\mathrm{AUC}_{0-24}$ of $25-40 \mathrm{mg} * \mathrm{~h} / \mathrm{L}$ in combination with QTc interval prolongation. In the case of very low moxifloxacin exposure $\left(\mathrm{AUC}_{0-24}<20 \mathrm{mg} * \mathrm{~h} / \mathrm{L}\right)$ in combination with a prolonged QTc interval, the physician will be advised to reconsider the anti-TB regimen as moxifloxacin may be less active than expected.

\section{Laboratory methods}

Drug analysis

Measurement of moxifloxacin and levofloxacin plasma/ serum concentrations will take place at the laboratory of the Department of Clinical Pharmacy and Pharmacology in the UMCG, The Netherlands, and using validated liquid chromatography-mass spectrometry methods. The method for levofloxacin has an accuracy of $0.1 \%-12.7 \%$, within-run precision of $1.4 \%-2.4 \%$ and between-run precision of $3.6 \%-4.1 \%$. The calibration curve is linear over a range of $0.10-5.00 \mathrm{mg} / \mathrm{L} .{ }^{25}$ This range was successfully expanded to $0.20-50.0 \mathrm{mg} / \mathrm{L}$ in a recent update of the method (data on file). Accuracy of the moxifloxacin method is $2.7 \%-7.1 \%$, within-run precision $1.4 \%-1.6 \%$ and between-run precision $1.0 \%-1.6 \%$. The calibration curve is linear over a range of $0.05-5.00 \mathrm{mg} / \mathrm{L} .{ }^{26}$ For both fluoroquinolones, only the total concentration (bound and unbound) will be measured. Therefore, the target $\mathrm{AUC}_{0-24} / \mathrm{MIC}$ values of $>150^{19}$ and $>100^{20}$ will be used for levofloxacin and moxifloxacin, respectively (table 2 ).

Plasma and serum samples containing levofloxacin are stable for at least 10 days at $50^{\circ} \mathrm{C}$ and can, therefore, be transported to the central facility in ambient temperature, without the need of transport on dry ice. ${ }^{27}$ The thermal stability of moxifloxacin was also tested according to the method of Ghimire et $a l$ and showed that moxifloxacin serum and plasma samples are stable for at least 10 days at $50^{\circ} \mathrm{C}$ as well (data on file).

\section{Microbiology}

The assessment of sputum smear and culture status after 2 months of MDR-TB treatment will be performed according to the local procedures, but at least once a month until documented culture conversion. MIC determination is preferred but not mandatory for TDM and will be performed according to local procedures as well. To account for the differences in culture media used in drug susceptibility testing, correction factors based on the critical concentrations in the WHO document 'Technical Report on critical concentrations for drug susceptibility testing of medicines used in the treatment of drugresistant tuberculosis' will be applied.$^{28}$ The target $\mathrm{AUC}_{0-}$ ${ }_{24} / \mathrm{MIC}$ values for each medium are shown in table 2. Furthermore, second-line molecular drug susceptibility tests will be considered in case MIC data are not available.

\section{Data analysis plan}

The primary outcome to assess the feasibility of centralised TDM will be the turn-around time, which is defined by the time between blood sampling and the peripheral centres receiving the TDM results including the dosing advice. The procedure is considered feasible if $>80 \%$ of the collected samples will be reported back to the physician within 7 days and $100 \%$ within 2 weeks. Additionally, the feasibility will be evaluated using secondary outcomes of sample quality after shipping and completeness of required information on the sample form.

Furthermore, we will evaluate the role of TDM on MDR-TB treatment by comparing the percentages of patients with sputum smear and culture conversion at 2 months in the enrolled groups. In addition, we will evaluate the number of patients with low fluoroquinolone exposure requiring dose changes after TDM to estimate the potential gains.

Table 2 Target $\mathrm{AUC}_{0-24} / \mathrm{MIC}$ and $\mathrm{AUC}_{0-24}$ for TDM of moxifloxacin and levofloxacin in patients with MDR-TB

\begin{tabular}{|c|c|c|c|c|c|}
\hline \multirow[b]{2}{*}{ Fluoroquinolone } & \multirow[b]{2}{*}{ Pulmonary MDR-TB } & \multicolumn{3}{|c|}{ Target $\mathrm{AUC}_{0-24} / \mathrm{MIC}^{*}$} & \multirow[b]{2}{*}{ Target AUC ${ }_{0-24}\left(\mathrm{mg}^{*} \mathrm{~h} / \mathrm{L}\right)$} \\
\hline & & MGIT & $7 \mathrm{H} 10 / 11$ & LJ & \\
\hline Moxifloxacin & Standard disease & $>100$ & $>50$ & $>25$ & $>40$ \\
\hline \multirow[t]{2}{*}{ Levofloxacin } & Standard disease & $>150$ & $>150 \ddagger$ & $>75$ & $>150$ \\
\hline & Severe disease or comorbidities & $>150$ & $>150 \ddagger$ & $>75$ & $>200 \dagger$ \\
\hline
\end{tabular}

Standard disease is defined as non-cavitary and regular disease on the radiograph. Severe disease is defined as cavitary or extensive disease on the radiograph.

${ }^{*}$ MIC varies depending on growth media; mycobacteria growth indicator tubes (MGIT), Middlebrook 7H10/7H11, and Lowenstein-Jensen (LJ) agar.

†Target $\mathrm{AUC}_{0-24} / \mathrm{MIC}$ at site of cavity; therefore, higher $\mathrm{AUC}_{0-24}$ is required.

†Levofloxacin critical concentration of $7 \mathrm{H} 11$ was extrapolated to $7 \mathrm{H} 10$.

AUC

, area under the concentration-time curve; LJ, Lowenstein-Jensen; MDR-TB, multidrug-resistant tuberculosis; MGIT, mycobacteria growth indicator tubes; MIC, minimal inhibitory concentration; TDM, therapeutic drug monitoring. 


\section{Sample size calculation}

As the primary endpoint was of descriptive nature and no data were available to perform a well-informed sample size calculation, it was decided to power the study on the clinical impact of TDM. The primary assumption was based on the detection of a proportional difference in sputum smear and culture positivity at 2 months of treatment in patients with MDR-TB undergoing TDM $(35 \%)^{29}$ and control patients $(60 \%) .{ }^{30}$ Given an alpha error of 0.05 and statistical power of $80 \%$, we calculated a sample size of 60 per single group (ie, 60 prospective and 120 historical control patients for moxifloxacin and equally for levofloxacin).

\section{PATIENT AND PUBLIC INVOLVEMENT}

There has been no patient or public involvement in the design of this study.

\section{ETHICS AND DISSEMINATION}

This study will be performed according to the Declaration of Helsinki and Good Clinical Practice. ${ }^{31}$ In each recruiting centre, ethical clearance has been granted according to local regulations and patient recruitment has begun at most sites (online supplementary file 1 ). Written informed consent will be obtained from all patients undergoing TDM. The need of new informed consent for historical controls was waived because of the use of retrospective anonymous data collected for programmatic purposes or previously reported data from studies for which patients had provided informed consent.

This study includes historical patients who did not receive TDM as controls instead of prospectively randomising patients to either receive or not receive TDM for ethical reasons. The evidence that TDM actually improves MDR-TB treatment outcomes has not been confirmed in randomised controlled trials, but multiple studies have described treatment failure and risk of antibiotic resistance due to subtherapeutic drug exposure of anti-TB drugs. ${ }^{8} 13151920$ In combination with a large between-patient pharmacokinetic variability, ${ }^{9} 10$ we hypothesise that TDM is able to improve treatment outcomes by ensuring adequate exposure in individual patients. Moreover, TDM for MDR-TB is recommended in guidelines when it is available. ${ }^{2} 3233$ We, therefore, considered it unethical to withhold TDM.

Study results will be published in a peer-reviewed journal and will be presented at an international conference.

\section{DISCUSSION}

We present an observational prospective multicentre study which aims to: (a) evaluate the feasibility of centralised TDM in differently resourced settings of varying TB endemicity and geographic region and (b) evaluate the role of TDM of moxifloxacin or levofloxacin on sputum smear and culture conversion rates in patients with MDR-TB after 2 months of treatment.

Presently, TDM is offered as an adjunctive to patients with TB in only a few hospitals worldwide and is considered to be part of the excellent clinical care. ${ }^{1623{ }^{23-36}}$ However, general interest in TDM and MDR-TB treatment optimisation has been increasing. A consensus statement on the diagnosis and treatment of MDR-TB in Europe states that TDM for second-line drugs should be used if available. ${ }^{33}$ Moreover, the use of second-line anti-TB drugs was listed in the American Thoracic Society guidelines as an indication for TDM and TDM is also recommended in the European Union Standards for Tuberculosis Prevention and Care ${ }^{32}{ }^{37}$ Yet, TDM is considered by some to be laborious, expensive and thus unpractical in countries with high TB incidence. Similar injurious arguments of economistic rationing of services were applied to second-line drugs for the treatment of MDR-TB in highly endemic settings and such rationing conversely led to amplification of the MDR-TB epidemic. ${ }^{38}$ This study will focus on the feasibility of centralised TDM, which could stimulate performing TDM more often as it requires only one qualified laboratory with validated analytical methods and devices in a central location. Other options to facilitate TDM are the implementation of LSSs, urine samples, dried-blood spots and saliva-screening methods. ${ }^{349-41}$ This study will additionally use LSSs to increase feasibility as well as to reduce the burden of TDM. The LSSs for moxifloxacin and levofloxacin used in this study $(0$ and 5 -hour postdose samples) were designed to optimise $\mathrm{AUC}_{0-24},{ }^{22}$ whereas the frequently used sampling schedule at 2-hour and 6-hour postdose is more suitable to estimate $\mathrm{C}_{\max }$ and identify delayed absorption. ${ }^{42}$

Although incorporating TDM in TB treatment has shown to give high treatment success rates in low endemic countries, such as The Netherlands, ${ }^{29}$ this has not yet been evaluated in well-designed randomised controlled trials. ${ }^{43}$ This study will provide a first-ever conclusion on the value of TDM of moxifloxacin and levofloxacin on sputum smear and culture conversion of patients with MDR-TB.

It can be considered a limitation that only TDM of fluoroquinolones is performed in this study. However, moxifloxacin and levofloxacin are currently among the core drugs in the MDR-treatment regimen together with linezolid and bedaquiline. ${ }^{3}$ Based on TDM criteria, ${ }^{44}$ we have selected moxifloxacin and levofloxacin because they show large interindividual pharmacokinetic variability, which emphasises the need for personalised dosing. ${ }^{9}{ }^{10}$ Moreover, fluoroquinolone resistance is on the rise and can develop during low drug exposure. ${ }^{8}$ TDM of fluoroquinolones aims to find the individual patients who have low drug exposure and would benefit from dose adjustment. Therefore, it is expected that the TDM of fluoroquinolones will have the largest impact on MDR-TB treatment outcomes. We did not include TDM for linezolid and bedaquiline in this study because of unclear evidence for TDM of bedaquiline due to the novelty of 
the drug ${ }^{45}$ and TDM of linezolid has focused more on preventing toxicity. ${ }^{46-48}$

Another limitation is that we are only evaluating interim outcomes such as sputum conversion rates at 2 months and will not assess outcomes at the end of treatment. However, this study is primarily designed to determine the feasibility of centralised TDM. In addition, this is the first study to evaluate the impact of fluoroquinolone TDM. We believe that reporting the results on sputum conversion rates is relevant as bacterial load and risk of acquired resistance are highest in the first months of therapy. Fast sputum culture conversion reduces the risk of transmission of M. tuberculosis strains which continues to sustain the MDR-TB epidemic. ${ }^{49}$ With the results of this study, we aim to design a future study to extensively evaluate TDM of all drugs in the regimen including the final treatment outcomes. However, such study would require substantial funding.

We hope that this study will show that centralised TDM is feasible and that TDM can improve the quality of treatment in terms of faster sputum conversion rates compared with historical experience. If that might be the case, the major hesitations about TDM in TB treatment can be attenuated favouring the improvement of TB management using a personalised approach. ${ }^{37}$

\section{Author affiliations}

${ }^{1}$ Department of Clinical Pharmacy and Pharmacology, University of Groningen, University Medical Center Groningen, Groningen, The Netherlands

${ }^{2}$ Department of Pulmonary Diseases and Tuberculosis, University of Groningen, University Medical Center Groningen, Groningen, The Netherlands

${ }^{3}$ Tuberculosis Center Beatrixoord, University of Groningen, University Medical Center Groningen, Haren, The Netherlands

${ }^{4}$ Department of Multidrug Resistant Tuberculosis, Riga East University Hospital TB and Lung Disease Clinic, Riga, Latvia

${ }^{5}$ Division of Infectious Diseases, Department of Medicine, Solna, Karolinska Institutet, Stockholm, Sweden

${ }^{6}$ Department of Infectious Diseases, Karolinska University Hospital, Stockholm, Sweden

${ }^{7}$ Department of Respiratory Medicine and Metro South Clinical Tuberculosis Service, Princess Alexandra Hospital, Woolloongabba, Queensland, Australia

${ }^{8}$ Division of Infectious Diseases and International Health, University of Virginia, Charlottesville, Virginia, USA

${ }^{9}$ The Republican Scientific and Practical Center for Pulmonology and Tuberculosis, Minsk, Belarus

${ }^{10}$ Department of Respiratory Medicine, Blizard Institute, Queen Mary University of London, Barts Health NHS Trust, London, UK

${ }^{11}$ Pulmonary Department, Respiratory Infections Unit, G. Papanikolaou Hospital, Aristotle University of Thessaloniki, Thessaloniki, Greece

${ }^{12}$ Drug-Resistant Tuberculosis Unit, 'Sotiria' Hospital for Chest Diseases, Athens, Greece

${ }^{13}$ Kibong'oto Infectious Diseases Hospital, Kilimanjaro, United Republic of Tanzania

${ }^{14}$ Clínica de Tuberculosis, Instituto Nacional de Enfermedades Respiratorias, Mexico City, Mexico

${ }^{15}$ Department of Medical, Surgical and Experimental Sciences, Clinical Epidemiology and Medical Statistics Unit, University of Sassari, Sassari, Italy

${ }^{16}$ Department of Medical and Surgical Sciences, Unit of Infectious Diseases, Alma Mater Studiorum University of Bologna, Bologna, Italy

${ }^{17}$ Department of Infection, Blizard Institute, Queen Mary University of London, Barts Health NHS Trust, London, UK

${ }^{18}$ Department of Internal Medicine, University of Groningen, University Medical Center Groningen, Groningen, The Netherlands

${ }^{19}$ Laboratory of Immunobiology and Genetics, Instituto Nacional de Enfermedades Respiratorias, Mexico City, Mexico
${ }^{20}$ Tecnologico de Monterrey, Escuela de Medicina y Ciencias de Salud, Mexico City, Mexico

${ }^{21}$ Servizio di Epidemiologia Clinica delle Malattie Respiratorie, Istituti Clinici Scientifici Maugeri IRCCS, Tradate, Italy

${ }^{22}$ Sydney Pharmacy School, Faculty of Medicine and Health, The University of Sydney, Sydney, New South Wales, Australia

Acknowledgements The project is part of the scientific activities of the Global Tuberculosis Network (GTN; Committees on Pharmacology: chair J-WA; Treatment: chair MMT and Global TB Consilium: chairs MT and ST) and of the WHO Collaborating Centre for Tuberculosis and Lung Diseases, Tradate, ITA-80, 2017-2020- GBM/RC/LDA.

Contributors SHJvdE, MGGS, DJT, GBM and J-WA designed the major outlines of the study. OA, LB, JB, GE, SKH, HH, LK, HK, JK, KM, CM, SGM, MMT, AS, GS, MT, ST, $\mathrm{FV}, \mathrm{TSvdW}, \mathrm{MRW}$ and JZ contributed to the study design. OA, LB, JB, GE, SKH, HH, LK, HK, JK, KM, CM, SGM, MMT, AS, MT, ST, FV, TSvdW, MRW and JZ will include patients in the study. GS performed the sample size calculation. SHJvdE wrote the first draft of the manuscript together with MGS, DJT, and J-WA. All authors read and approved the final version of the manuscript.

Funding The authors have not declared a specific grant for this research from any funding agency in the public, commercial or not-for-profit sectors.

Competing interests None declared.

Patient and public involvement Patients and/or the public were not involved in the design, or conduct, or reporting, or dissemination plans of this research.

Patient consent for publication Not required.

Provenance and peer review Not commissioned; externally peer reviewed.

Open access This is an open access article distributed in accordance with the Creative Commons Attribution Non Commercial (CC BY-NC 4.0) license, which permits others to distribute, remix, adapt, build upon this work non-commercially, and license their derivative works on different terms, provided the original work is properly cited, appropriate credit is given, any changes made indicated, and the use is non-commercial. See: http://creativecommons.org/licenses/by-nc/4.0/.

ORCID iD

Jan-Willem Alffenaar http://orcid.org/0000-0001-6703-0288

\section{REFERENCES}

1 World Health Organization. Global tuberculosis report, 2018.

2 World Health Organization. WHO consolidated guidelines on drugresistant tuberculosis treatment, 2019.

3 World Health Organization. Rapid communication: key changes to treatment of multidrug- and rifampicin-resistant tuberculosis (MDR/ RR-TB), 2018.

4 Collaborative Group for the Meta-Analysis of Individual Patient Data in MDR-TB treatment-2017, Ahmad N, Ahuja SD, et al. Treatment correlates of successful outcomes in pulmonary multidrug-resistant tuberculosis: an individual patient data meta-analysis. Lancet 2018;392:821-34.

5 Alffenaar J-WC, Migliori GB, Gumbo T. Multidrug-resistant tuberculosis: pharmacokinetic and pharmacodynamic science. Lancet Infect Dis 2017:17:898.

6 World Health Organization. Global tuberculosis report, 2012.

7 Davies Forsman L, Bruchfeld J, Alffenaar J-WC. Therapeutic drug monitoring to prevent acquired drug resistance of fluoroquinolones in the treatment of tuberculosis. Eur Respir J 2017;49:1700173.

8 Srivastava S, Pasipanodya JG, Meek C, et al. Multidrug-resistant tuberculosis not due to noncompliance but to between-patient pharmacokinetic variability. $J$ Infect Dis 2011;204:1951-9.

9 Van't Boveneind-Vrubleuskaya N, Seuruk T, van Hateren K, et al. Pharmacokinetics of levofloxacin in multidrug- and extensively drug-resistant tuberculosis patients. Antimicrob Agents Chemother 2017;61:e00343-17.

10 Pranger AD, van Altena R, Aarnoutse RE, et al. Evaluation of moxifloxacin for the treatment of tuberculosis: 3 years of experience. Eur Respir J 2011;38:888-94.

11 Ghimire S, Van't Boveneind-Vrubleuskaya N, Akkerman OW, et al. Pharmacokinetic/pharmacodynamic-based optimization of levofloxacin administration in the treatment of MDR-TB. J Antimicrob Chemother 2016;71:2691-703.

12 Alffenaar J-WC, Gumbo T, Aarnoutse RE. Acquired drug resistance: we can do more than we think! Clin Infect Dis 2015;60:969-70. 
13 Pasipanodya JG, Mcllleron H, Burger A, et al. Serum drug concentrations predictive of pulmonary tuberculosis outcomes. $J$ Infect Dis 2013;208:1464-73.

14 Modongo C, Pasipanodya JG, Magazi BT, et al. Artificial intelligence and amikacin exposures predictive of outcomes in multidrugresistant tuberculosis patients. Antimicrob Agents Chemother 2016;60:5928-32.

15 Sekaggya-Wiltshire C, von Braun A, Lamorde M, et al. Delayed sputum culture conversion in tuberculosis-human immunodeficiency virus-coinfected patients with low isoniazid and rifampicin concentrations. Clin Infect Dis 2018;67:708-16.

16 Alffenaar J-WC, Tiberi S, Verbeeck RK, et al. Therapeutic drug monitoring in tuberculosis: practical application for physicians. Clin Infect Dis 2017;64:104-5.

17 Peloquin $\mathrm{C}$. The role of therapeutic drug monitoring in mycobacterial infections. Microbiol Spectr 2017;5:TNMI7-00295-2016.

18 Alkabab Y, Keller S, Dodge D, et al. Early interventions for diabetes related tuberculosis associate with hastened sputum microbiological clearance in Virginia, USA. BMC Infect Dis 2017;17:125.

19 Deshpande D, Pasipanodya JG, Mpagama SG, et al. Levofloxacin pharmacokinetics/pharmacodynamics, dosing, susceptibility breakpoints, and artificial intelligence in the treatment of multidrugresistant tuberculosis. Clin Infect Dis 2018;67:S293-302.

20 Gumbo T, Louie A, Deziel MR, et al. Selection of a moxifloxacin dose that suppresses drug resistance in mycobacterium tuberculosis, by use of an in vitro pharmacodynamic infection model and mathematical modeling. $J$ Infect Dis 2004;190:1642-51.

21 van den Elsen SHJ, Sturkenboom MGG, Van't BoveneindVrubleuskaya $\mathrm{N}$, et al. Population pharmacokinetic model and limited sampling strategies for personalized dosing of levofloxacin in tuberculosis patients. Antimicrob Agents Chemother 2018;62:e01092-18.

22 van den Elsen SHJ, Sturkenboom MGG, Akkerman OW, et al. Limited sampling strategies using linear regression and the bayesian approach for therapeutic drug monitoring of moxifloxacin in tuberculosis patients. Antimicrob Agents Chemother 2019;63:e00384-19.

23 Ghimire S, Bolhuis MS, Sturkenboom MGG, et al. Incorporating therapeutic drug monitoring into the world health organization hierarchy of tuberculosis diagnostics. Eur Respir J 2016;47:1867-9.

24 Schimke I. Quality and timeliness in medical laboratory testing. Anal Bioanal Chem 2009;393:1499-504.

25 Ghimire S, van Hateren K, Vrubleuskaya N, et al. Determination of levofloxacin in human serum using liquid chromatography tandem mass spectrometry. J Appl Bioanal 2018;4:16-25.

26 Pranger AD, Alffenaar J-WC, Wessels AMA, et al. Determination of moxifloxacin in human plasma, plasma ultrafiltrate, and cerebrospinal fluid by a rapid and simple liquid chromatography- tandem mass spectrometry method. J Anal Toxicol 2010;34:135-41.

27 Ghimire S, Jongedijk EM, van den Elsen SHJ, et al. Cross validation of liquid chromatography tandem mass spectrometry method for quantification of levofloxacin in saliva.

28 World Health Organization. Technical report on critical concentrations for drug susceptibility testing of medicines used in the treatment of drug-resistant tuberculosis, 2018.

29 van Altena R, de Vries G, Haar $\mathrm{CH}$, et al. Highly successful treatment outcome of multidrug-resistant tuberculosis in the Netherlands, 2000-2009. Int J Tuberc Lung Dis 2015;19:406-12.

30 Sotgiu G, Centis R, D'Ambrosio L, et al. Efficacy, safety and tolerability of linezolid containing regimens in treating MDR-TB and XDR-TB: systematic review and meta-analysis. Eur Respir J 2012;40:1430-42.
31 World Medical Association. World medical association declaration of Helsinki: ethical principles for medical research involving human subjects. JAMA 2013;310:2191-4.

32 Nahid P, Dorman SE, Alipanah N, et al. Official American thoracic society/centers for disease control and prevention/infectious diseases society of America clinical practice guidelines: treatment of drug-susceptible tuberculosis. Clin Infect Dis 2016;63:e147-95.

33 Lange C, Abubakar I, Alffenaar J-WC, et al. Management of patients with multidrug-resistant/extensively drug-resistant tuberculosis in Europe: a TBNET consensus statement. Eur Respir J 2014;44:23-63.

34 Alffenaar J-WC, Heysell SK, Mpagama SG. Therapeutic drug monitoring: the need for practical guidance. Clin Infect Dis 2019;68:1065-6.

35 Lange C, Dheda K, Chesov D, et al. Management of drug-resistant tuberculosis. Lancet 2019;394:953-66.

36 Alffenaar J-WC, Gumbo T, Dooley KE, et al. Integrating pharmacokinetics and pharmacodynamics in operational research to end tuberculosis. Clin Infect Dis 2020;70:1774-80.

37 Migliori GB, Sotgiu G, Rosales-Klintz S, et al. ERS/ECDC statement: European union standards for tuberculosis care, 2017 update. Eur Respir J 2018;51:1702678.

38 Nicholson T, Admay C, Shakow A, et al. Double standards in global health: medicine, human rights law and multidrug-resistant TB treatment policy. Health Hum Rights 2016;18:85-102.

39 Ghimire S, Maharjan B, Jongedijk EM, et al. Evaluation of saliva as a potential alternative sampling matrix for therapeutic drug monitoring of levofloxacin in patients with multidrug-resistant tuberculosis. Antimicrob Agents Chemother 2019;63:e02379-18.

40 Zuur MA, Bolhuis MS, Anthony R, et al. Current status and opportunities for therapeutic drug monitoring in the treatment of tuberculosis. Expert Opin Drug Metab Toxicol 2016;12:509-21.

41 Zentner I, Modongo C, Zetola NM, et al. Urine colorimetry for therapeutic drug monitoring of pyrazinamide during tuberculosis treatment. Int J Infect Dis 2018;68:18-23.

42 Lange C, Aarnoutse RE, Alffenaar JWC, et al. Management of patients with multidrug-resistant tuberculosis. Int J Tuberc Lung Dis 2019;23:645-62.

43 van der Burgt EPM, Sturkenboom MGG, Bolhuis MS, et al. End TB with precision treatment! Eur Respir J 2016;47:680-2.

44 Figueras A. WHO report 'review of the evidence to include TDM in the essential in vitro diagnostics list and prioritization of medicines to be monitored', 2019.

45 Borisov SE, Dheda K, Enwerem M, et al. Effectiveness and safety of bedaquiline-containing regimens in the treatment of MDR- and XDRTB: a multicentre study. Eur Respir J 2017;49:1700387.

46 Bolhuis MS, Tiberi S, Sotgiu G, et al. Is there still room for therapeutic drug monitoring of linezolid in patients with tuberculosis? Eur Respir J 2016;47:1288-90.

47 Alffenaar J-WC, van Altena R, Harmelink IM, et al. Comparison of the pharmacokinetics of two dosage regimens of linezolid in multidrugresistant and extensively drug-resistant tuberculosis patients. Clin Pharmacokinet 2010;49:559-65.

48 Bolhuis MS, Akkerman OW, Sturkenboom MGG, et al. Linezolidbased regimens for multidrug-resistant tuberculosis (TB): a systematic review to establish or revise the current recommended dose for TB treatment. Clin Infect Dis 2018;67:S327-35.

49 Migliori GB, Nardell E, Yedilbayev A, et al. Reducing tuberculosis transmission: a consensus document from the world health organization regional office for Europe. Eur Respir $J$ 2019;53:1900391. 